\section{OP0142 FAMILY PAIN HISTORY PREDICTS CHILD HEALTH STATUS IN CHILDREN WITH CHRONIC RHEUMATIC DISEASE}

LE Schanberg, KK Anthony, KM Gil, JC Lefebvre, DW Kredich, LM Macharoni. 'Duke University Medical Centre, Durham, NC, USA; ${ }^{2}$ UNC, Chapel Hill, NC, USA; ${ }^{3}$ Wofford College, Spartanburg, SC, USA

\subsection{6/annrheumdis-2001.75}

Objectives To examine the relationships between parental and family pain history and the pain report and physician-rated health status of children with chronic rheumatic disease, and to determine whether child coping strategy mediates the relationship between family pain history and the child's pain and physician-rated health status.

Method Parents of 100 children were recruited from a paediatric rheumatology clinic during routine visits. Parents completed questionnaires assessing parental pain history and family characteristics. Children in the study completed a series of questionnaires to assess pain and pain coping strategies. A paediatric rheumatologist provided a global assessment of disease severity as an index of child health status.

Results A high number of parents of children seen in a paediatric rheumatology clinic described a personal pain history. Over $90 \%$ of parents reported having at least one chronic pain condition, with an equal proportion reporting an episode of pain in the past month. Correlational analyses indicated that parents reporting higher levels of current pain and higher mean levels of pain during the past month were more likely to have children reporting higher levels of current pain. In addition, parents who sought more treatment for their own pain and reported higher levels of pain-related interference with activity were more likely to have children reporting higher levels of pain and presenting with poorer health status. Finally, a series of mediational statistical models confirmed that child use of the pain coping strategy, catastrophizing, mediated the relationship between several parent and family pain history variables and the child's own current pain ratings and physician global assessment.

Conclusions The results from the present study highlight potentially important relationships between parent and child pain experiences and suggest that parents should participate in interventions aimed at improving children's abilities to cope with pain.

\section{OP0143 GENETIC DETERMINANTS OF THE TH1 BIAS IN RHEUMATOID ARTHRITIS}

A Skapenko, J Wendler, R Mueller, PE Lipsky, JR Kalden, H Schulze-Koops. 'Nikolaus Fiebiger Center for Molecular Medicine, Clinical Research Group III, and Department of Internal Medicine III and Institue for Clinical Immunology, University of Erlangen-Nuremberg, Erlangen, Germany; ${ }^{2}$ National Institute of Arthritis and Musculoskeletal and Skin Diseases, Rockville Pike, Bethesda, MD, USA

\subsection{6/annrheumdis-2001.76}

Rheumatoid arthritis (RA) is a chronic systemic inflammatory disease, which is likely to be driven by activated pro-inflammatory Th1 cells. In contrast, Th2 cells and their cytokines, in particular IL-4 are rarely found in RA. It could recently be demonstrated that memory $\mathrm{T}$ cells from patients with early $\mathrm{RA}$ manifest a profound functional deficit in that they cannot differentiate into Th2 cells even at very early stages of the disease. The inability of memory $\mathrm{T}$ cells to mount immunomodulatory
Th2 effectors might contribute to the Th1 bias in RA and, thus, might by critically involved in the initiation of chronic autoimmune inflammation. The mechanisms, however, involved in this abnormality have not been delineated in detail. We have recently established a system to analyse $\mathrm{T}$ cell differentiation in vitro and have identified molecular mechanisms regulating Th2 effector generation in healthy individuals. In the current proposal, molecular mechanisms will be identified that are involved in the development of Th1 mediated immunity in patients with very early rheumatoid arthritis (symptoms at time of blood collection of $\leq 2$ weeks) using gene chip microarray technology. Selected candidate genes associated with the Th2 cell differentiation defect will be tested for their functional significance in biological assays. The definition of mechanisms contributing to the Th1 differentiation bias in very early RA will provide important insights into the pathogenesis of the disease, in particular into the initial steps of the evolving immune response, and might lead to the development of novel therapeutic strategies.

\section{OP0144 DEVELOPMENT OF A PROSPECTIVE, LONGITUDINAL, INCEPTION COHORT TO EVALUATE THE OUTCOME OF RHEUMATOID ARTHRITIS IN AFRICAN-AMERICANS}

R Brasington. Washington University School of Medicine, St. Louis, MO, USA

10.1136/annrheumdis-2001.77

It has been established that the prevalence of rheumatoid arthritis (RA) in African-Americans (AA) in the United States is comparable to that of the white population. However, data comparing the clinical manifestations and outcome of RA in AA and other ethnic groups is limited. Approximately $90 \%$ of the AA population of St. Louis, MO. lives within an area of twelve contiguous zip codes, within which is the Washington University School of Medicine (WUSM). Almost $60 \%$ of the individuals within these zip codes have incomes below the poverty level, and the vast majority of such patients receive their medical care at WUSM Rheumatology Clinic. In addition, our clinic provides care for patients from the surrounding geographic area who have a more diverse distribution of income and insurance status.

We propose to develop an inception cohort of AA patients with RA by ACR criteria to be entered within one year of diagnosis, and follow this cohort prospectively in a longitudinal fashion. The primary comparison group will be white patients with early RA seen at the same outpatient clinic; Russian immigrants, Hispanics, and Asian-Pacific rim patients will serve as additional comparison groups if numbers of patients are sufficient. Demographic data will be collected on zip code, income, education, medical insurance status, and literacy. Treatment for each patient will be non-randomised, and determined by the patient's treating rheumatologist. Outcome measures will include the ACR core data set (tender and swollen joint count, MD and patient global assessment, pain VAS by patient, HAQ, and ESR), to be collected at baseline and every six months. Sharp scores will be obtained on radiographs of hands in all patients at baseline and at yearly intervals.

Data will be analysed to compare ACR 50, ACR 70, mean ACR (N), HAQ scores, and Sharp scores for each group. We will also compare use of Disease Modifying Antirheumatic Drugs (DMARDs), specifically with regard to initiation in the first year, use of combinations of DMARDs, and use of biologic agents. Multivariate regression analysis will be performed to adjust for confounding variables which might influence outcome, such as 
income, education, duration of symptoms before seeking medical treatment, duration of symptoms before the introduction of DMARDs, and specific DMARDs used.

\section{OP0145 GENE-ENVIRONMENT AND DISEASE INTERACTIONS: IS IT SMART TO USE COHORT STUDIES?}

TWJ Huizinga, M Kloppenburg. Department of Rheumatology and Epidemiology, Leiden University Medical Center, Leiden, The Netherlands

10.1136/annrheumdis-2001.78

Although much progress has been made in understanding the pathophysiology of RA, very few data are present on the cause of the disease. During the last years genetic factors (HLA, the X -chromosome) and environmental factors (smoking) have been identified as factors that are more often present in RA patients than in controls. By using a cohort study that enrols all patients with arthritis of less than two years of disease duration (The Leiden Early Arthritis Clinic), we were able to identify additional risk factors to develop RA such as a reduced fecundity in RA patients. However the current methodology to identify risk factors can be questioned. This presentation will focus on the alternative techniques such as case-case series or case-control studies as a more efficient approach to identify risk factors to develop RA.

\section{Infectious trigger mechanisms in R. D. - Thursday June 14, 10.00-11.45/Forum Hall}

\section{SP0015 THE DETECTION OF MICROBIAL DNA IN THE JOINT}

J Gaston. Rheumatology, University of Cambridge, Cambridge, UK

10.1136/annrheumdis-2001.79

Lyme disease, Whipple's disease and reactive arthritis are clear examples of forms of arthritis caused by bacterial infection, but the idea that infection might be involved in diseases like rheumatoid arthritis (RA) has a long history. Although joints in all of these conditions are usually "sterile" (i.e. no bacteria can be cultured from the joint), molecular methods of detection have demonstrated the presence of bacterial nucleic acids. The fact that ribosomal RNA (rRNA) genes from all bacteria have highly conserved sequences interspersed with species-specific sequences makes it possible to use polymerase chain reaction (PCR) techniques to detect any bacteria present in synovium and synovial fluid by amplification using "universal" primers which identify the conserved sequences, and then sequencing the products to determine the bacteria from which they arises. When this technique has been applied to synovium from RA patients, multiple species of bacteria have been identified, both by PCR (showing the presence of bacterial DNA) and RT-PCR; the latter implies the presence of live bacteria since bacterial rRNA is unstable, unlike DNA. These results cannot be accounted for by artefactual contamination, and suggest that resident bacteria such as gut and skin-derived organisms can find their way to the joint, most probably within phagocytes which have previously engulfed them and then been recruited to the inflamed joint. The same results were obtained when synovial fluid from reactive arthritis patients was tested. However in reactive arthritis, by using species-specific primers, it was also possible to detect the arthritistriggering organism (Chlamydia, Yersinia) in a proportion of cases. The specific organism was not detected simply by sequencing the product obtained using universal primers, which suggests that the specific organism is a minor component of the total bacterial colonisation of the joint.

Bacterial products such as DNA, LPS and protein antigens may contribute to joint inflammation both as targets of specific immune responses and as adjuvants. Nevertheless, reactive arthritis and RA differ in that the former usually resolves spontaneously; thus the presence of bacteria is not sufficient to cause chronic inflammation and other mechanisms which engender chronicity must be important in RA. Against a background of commensal bacteria it may be difficult to detect specific diseaseassociated organisms in RA, since specific organisms are in a minority in the joint even in diseases such as reactive arthritis in which they are clearly important in pathogenesis.

\section{SP0016 THE SPONDYLOARTHROPATHIES AS A PARADIGM FOR THE INDUCTION OF AN AUTOIMMUNE RESPONSE BY BACTERIA}

J Sieper. Rheumatology, University Hospital Benjamin Franklin and German Rheumatology Research Center, Berlin, Germany

10.1136/annrheumdis-2001.80

The spondyloarthropathies (SpA) comprise ankylosing spondylitis (AS), reactive arthritis (ReA) or Reiter's syndrome, arthritis/ spondylitis with inflammatory bowel disease (IBD) and arthritis/ spondylitis with psoriasis. The main link between each other is the association with HLA-B27, the same pattern of peripheral joint involvement with an asymmetrical arthritis predominantly of the lower limbs, and the possible occurrence of sacroiliitis, spondylitis, enthesitis and uveitis. Bacteria play a central role in the pathogenesis of the SpA. This is most obvious in ReA which is triggered by preceding bacterial infections of the urogenital or gastroenteral tract. In arthritis/spondylitis associated with IBD a causative role of gut bacteria is also assumed which can stimulate the cellular immune sytem because the breakage of the gut barrier. Because between $20-50 \%$ of HLA-B27-positive patients with ReA or IBD move on to AS a causative or triggering role of bacteria can also be assumed in AS. Due to more recent studies using new imaging techniques such as MRI and immunohistological investigations it can now be assumed that the immunopathology of SpA occurs typically at the interphase of bone and cartilage and that synovitis is only a secondary event. Although at the moment it cannot be excluded that bacteria or pieces of bacteria persist in these structures this seems to be unlikely. Thus, an autoimmune response triggered by a bacterial infection seems to be more likely. Cartilage derived proteins such as aggrecan could be, according to studies in animal models of SpA and to studies in SpA patients, a primary target of this autoimmune response. Such a response could be due to cross-reactivity with a bacterial antigen. This possible course of events leading to a chronic immune response in SpA has to be proven in the near future. The identification of target antigens would offer the possibility to switch off the antigen-specific immune response as a new exciting treatment option. 